\title{
Otimização da Formulação de uma Mistura Parte 2: Custo
}

\author{
José Luis Duarte Ribeiro
}

Doutor em Engenharia - PPGEP/UFRGS

Giovana Savitri Pasa

Mestranda do PPGEP/UFRGS

Danielle Barcos Nunes

Mestranda do PPGEP/UFRGS

Programa de Pós-Graduação em Engenharia de Produção - UFRGS

Praça Argentina no. 9 - Porto Alegre, RS - 90040-020

Palavras-Chave: Qualidade, Custos de Produção, Otimização, Formulações
Quimicas

Key words: Quality, Production Costs, Optimization, Chemical Mixture.

\section{RESUMO}

Este artigo é a continuação de um trabalho anterior no qual foi apresentado um estudo de caso visando a otimização da formulação de uma mistura de borracha. No estudo anterior a otimização foi feita considerando exclusivamente o aspecto qualidade. O presente artigo estende o que foi feito anteriormente em vários aspectos: (i) os custos devido à má qualidade são expressos em unidades monetárias, (ii) os custos de produção são modelados, (iii) uma função de custos globais é apresentada, e (iv) é feito um estudo de otimização que define o ajuste dos fatores controláveis (componentes da mistura) que leva a minimização dos custos globais. Além de apresentar um envelope operacional que circunda o ótimo global, o método utilizado permite quantificar as parcelas que contribuem para o custo total da mistura. Dessa forma, caso por motivos estratégicos seja necessário abandonar o ótimo global, para obter uma redução no custo de produção, ou uma melhoria na qualidade, os modelos apresentados indicam qual o caminho a seguir.

\section{ABSTRACT}

This paper is the continuation of a previous one where a case study dealing with the optimization of a chemical compound was presented. In the previous paper the optimization was accomplished taken into account only quality. The present paper expands what was done previously in several ways: (i) the costs due to poor quality are expressed in monetary units, (ii) production costs are modeled, (iii) a global cost function is presented, and (iv) an optimization study which defines the best set for the control factors (quantities of mixture components) is performed. Besides presenting an operational window which contains the global optimum, the applied method allows one to quantify the terms that contribute to the mixture total cost. Thus, if for strategic reasons the global optimum has to be abandoned (e.g., to reduce production costs or to maximize quality alone), the models presented indicate the way to follow.

Belo Horizonte, Vol 7, N², p.189-201

Nov. 1997 


\section{PRODUÇÃO}

\section{Introdução}

Pode-se dizer que os custos globais de um produto se dividem em duas parcelas principais: uma parcela que independe dos ajustes operacionais, e compreende toda a classe de custos administrativos, e outra parcela que depende dos ajustes operacionais.

Este artigo discute os custos que dependem dos ajustes operacionais, os quais, por sua vez, podem ser subdivididos em uma parcela que será chamada de custos devido à má qualidade, e outra parcela que será chamada de custos de produção.

\section{Custos devido à má qualidade}

Um produto pode ter sua qualidade avaliada através de várias características de qualidade, as quais representam os aspectos importantes associados ao desempenho deste produto. Por exemplo, no caso da borracha, as características de qualidade de interesse são a Densidade, a Dureza, a Abrasão, etc. Estas Caracteristicas de Qualidade devem assumir os valores alvo especificados para elas, de forma a atenderem às expectativas dos clientes. À medida em que estas características se afastam de seu valor alvo, geram uma perda para o cliente, ou seja, um custo devido à má qualidade.

Uma alternativa para modelar os custos devido à má qualidade é o emprego da Função de Perda proposta por Ribeiro e Elsayed $(1993,1995)$, a qual busca avaliar a perda decorrente dos desvios que as diversas Características de Qualidade de interesse apresentam em relação aos seus valores ideais. Essa função de perda, que aparece descrita em detalhe em Pasa, Ribeiro e Nunes (1996), considera o peso que o cliente atribui a cada Caracteristica de Qualidade e é constituída de três parcelas.

A primeira parcela diz respeito à perda decorrente da diferença entre o valor alvo especificado para uma dada característica de qualidade do produto e o valor médio efetivamente obtido a partir do processo produtivo. Considera-se que a perda é diretamente proporcional ao quadrado desta diferença.

A segunda parcela diz respeito à variabilidade da característica de qualidade, observada quando se avaliam várias unidades do produto geradas com um mesmo ajuste dos fatores controláveis. Considera-se que a perda é diretamente proporcional à variância dos valores obtidos para a característica de saída do produto.

A terceira parcela diz respeito à sensibilidade das características de qualidade do produto à imprecisão dos ajustes nos fatores controláveis. Considera-se que a perda é diretamente proporcional ao produto da variabilidade de um certo fator controlável pela sensibilidade da característica de qualidade a esta variabilidade.

\section{Custos de produção}

O termo custos de produção será utilizado neste trabalho para representar aqueles custos que dependem dos ajustes 
operacionais. No caso da otimização de uma formulação, esses ajustes operacionais são os percentuais dos componentes que fazem parte da formulação. Alterando esses percentuais, altera-se o custo da mistura, uma vez que os custos dos componentes individuais não são idênticos.

A modelagem dos custos de produção em geral é simples, uma vez que depende diretamente dos custos das matérias primas. Em geral, um modelo determinístico pode ser usado, a partir dos dados obtidos junto aos técnicos da empresa ou junto aos fornecedores.

\section{Objetivos deste trabalho}

Este trabalho apresenta a otimização da formulação de uma mistura de borracha, considerando simultaneamente os custos devido à má qualidade e os custos de produção. Ele complementa um estudo anterior descrito em Pasa, Ribeiro e Nunes, 1996.

No estudo descrito por Pasa, Ribeiro e Nunes (1996), o objetivo era modelar o efeito dos fatores controláveis, ou seja, das proporções dos ingredientes que compõem a mistura, sobre as características de qualidade de interesse e determinar quais os níveis ótimos destes fatores, considerando exclusivamente os aspectos relacionados à qualidade do produto final. Neste, são determinados os níveis ótimos dos fatores controláveis (composição da mistura), de forma a conciliar qualidade e custos de produção.

\section{Apresentação do Estudo de Caso}

A formulação em estudo constitui-se de uma mistura de borracha composta por vários ingredientes, dos quais quatro foram escolhidos como fatores controláveis, e seus efeitos sobre as características de qualidade foram investigados. Esses ingredientes serão designados por $\mathrm{X} 1, \mathrm{X} 2, \mathrm{X} 3 \mathrm{e} \mathrm{X} 4$; os demais ingredientes que compõem a mistura foram mantidos constantes durante todo o estudo.

As características de qualidade (CQ) consideradas na otimização da formulação foram seis: Abrasão, Densidade, Dureza, Tensão de Ruptura, Módulo 300\% e Alongamento. As mesmas serão referidas como Y1, Y2, .., Y6 (não necessariamente na mesma ordem em que foram citadas, para preservar a confidencialidade dos dados).

Conforme os respectivos valores alvo e limites de especificação, classificou-se cada CQ como do tipo "maior-é-melhor" (quando possui apenas limite inferior de especificação), "menor-é-melhor" (apenas limite superior de especificação) ou "nominal-é-melhor" (ambos). Além disso, de acordo com a experiência dos engenheiros responsáveis, a cada CQ foi atribuído um peso, correspondente à sua importância relativa na avaliação da qualidade global da mistura. Esses valores são apresentados na Tabela 1 a seguir. 


\section{PRODUÇÃO}

\begin{tabular}{llllll}
\hline CQ & $\begin{array}{l}\text { Importincia } \\
\text { Relativa }\end{array}$ & Tipo & Alvo & $\begin{array}{l}\text { Limites } \\
\text { Mínimo }\end{array}$ & $\begin{array}{c}\text { Especificação } \\
\text { Máximo }\end{array}$ \\
\hline Y1 & 2,25 & menor-e-melhor & 111 & - & 113 \\
Y3 & 1,75 & nominal-e-melhor & 68 & 63 & 70 \\
Y4 & 1,50 & maior-é-melhor & 320 & 270 & - \\
Y5 & 1,00 & maior-é-melhor & 110 & 90 & - \\
Y6 & 1,25 & maior-é-methor & 110 & 90 & - \\
& 2,00 & menor-e-melhor & 180 & - & 230 \\
\hline
\end{tabular}

Tabela 1 - Características de Qualidade (CQ).

Na primeira parte deste estudo, apresentada por Pasa, Ribeiro e Nunes (1996), utilizaram-se técnicas de Projetos de Experimentos com Misturas para o planejamento da coleta de dados. $\mathrm{Na}$ Tabela 2, são apresentadas as faixas de variação percentual possível para cada um dos fatores controláveis, dentro das quais foram planejados 20 ensaios (misturas com diferentes composições).
Em cada ensaio, foram coletados dados sobre os valores resultantes das características de qualidade mencionadas anteriormente. A seguir, através de Análise de Regressão Múltipla, obtiveram-se modelos matemáticos capazes de estimarem o valor de cada uma das Características de Qualidade de interesse em função do ajuste dos fatores controláveis. Após, utilizou-se a Função

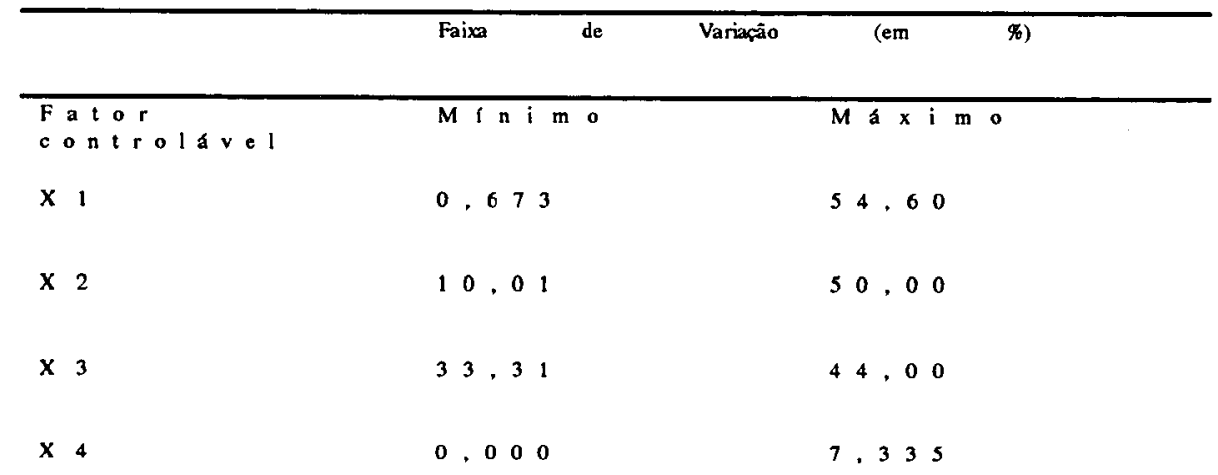

Tabela 2 - Faixas de variação possível dos fatores controláveis. 
de Perda proposta por Ribeiro e Elsayed (1995) para encontrar o ajuste ótimo para a qualidade global do produto.

Esta segunda parte do trabalho dá continuidade a anterior, apresentando a modelagem dos custos que dependem dos ajustes operacionais. No caso em questão, que trata da otimização de uma mistura, os ajustes operacionais são as quantidades dos componentes da mistura. A seguir, serão descritas as etapas principais da modelagem dos custos:

- Obtenção da Função de Perda em unidades monetárias:

Inicialmente, transformou-se a Função de Perda obtida no trabalho anterior para que esta passasse a estimar a perda diretamente em unidades monetárias. Obteve-se, assim, uma estimativa dos custos decorrentes da má qualidade do produto.

- Obtenção de um modelo para 0 custo de produção:

Os dados referentes aos custos de matéria prima fornecidos pelos engenheiros da empresa foram analisados, obtendo-se um modelo determinístico capaz de indicar o custo de produção da mistura em função de sua composição (niveis dos fatores controláveis).

\section{- Composição da função de custos} global:

Compôs-se, a seguir, uma função de custos global, a qual associa, numa única expressão matemática, os custos decorrentes da má qualidade e os custos de produção.

\section{PRODUÇÃO}

mistura:

\section{- Otimização do custo global da}

A partir da função de custo global da mistura, determinaram-se os niveis ótimos dos fatores controláveis, ou seja, a composição da mistura que minimizava o custo global.

\section{Modelagem dos Custos Dependentes dos Ajustes Operacionais}

\subsection{Custos Devidos à Má Qualidade}

Para aplicar a Função de Perda multivariada ao processo em questão, utilizaram-se as informações contidas nas Tabelas 1 e 2 e os modelos encontrados para as estimativas da média e da variabilidade de cada uma das características de qualidade (Tabela 3). Neste estudo, a variabilidade dos fatores controláveis (percentuais de $\mathrm{X} 1, \mathrm{X} 2, \mathrm{X} 3$ e X4 na mistura) foi considerada desprezível, não sendo necessário, então, estimar a sensibilidade.

É importante observar que todos os modelos apresentados a seguir, bem como a Função de Perda resultante, estão expressos, não em função dos percentuais dos fatores controláveis (X1, X2, X3 e $X 4)$, os quais apresentam uma relação de dependência linear entre si $(\mathrm{SXi}=100 \%)$, mas sim em função das variáveis independentes W (W1, W2 e W3). As transformações que permitem a passagem de um sistema de coordenadas para o outro são sugeridas por Cornell (1981) e encontram-se descritas em Pasa, Ribeiro e Nunes (1996). 


\section{PRODUÇÃO}

\begin{tabular}{|l|l|}
\hline \multicolumn{1}{|c|}{ Média } & Variabilidade \\
\hline $\mathrm{Y} 1=113,6-0,5651 \times \mathrm{W} 1+2,972 \times$ & $\mathrm{S} 1=0,1236$ \\
$\mathrm{~W} 2+0,2945 \times \mathrm{W} 3+0,212 \times \mathrm{W} 22$ & \\
$\mathrm{Y} 2=64,9+2,109 \times \mathrm{W} 1+5,881 \times$ & $\mathrm{S} 2=0,7529$ \\
$\mathrm{~W} 2-1,511 \times \mathrm{W} 3$ & \\
$\mathrm{Y} 3=315,4-8,458 \times \mathrm{W} 1+8,75 \times$ & $\mathrm{S} 3=11,96$ \\
$\mathrm{~W} 3+23,78 \times \mathrm{W} 32$ & \\
$\mathrm{Y} 4=159,65+24,66 \times \mathrm{W} 1+67,51$ & $\mathrm{~S} 4=8,80$ \\
$\times \mathrm{W} 2-14,94 \times \mathrm{W} 3+9,979 \times \mathrm{W} 12$ & \\
$+14,06 \times \mathrm{W} 22$ & \\
$\mathrm{Y} 5=93,3-11,03 \times \mathrm{W} 1-17,84 \times \mathrm{W} 2$ \\
$+6,668 \times \mathrm{W} 3-3,642 \times \mathrm{W} 12+5,572$ & $\mathrm{~S} 5=3,804$ \\
$\times \mathrm{W} 32$ & \\
$\mathrm{Y} 6=182,2-14,176 \times \mathrm{W} 1+11,252$ & $\mathrm{~S} 6=2,75$ \\
$\times \mathrm{W} 3-4,990 \times \mathrm{W} 1 \times \mathrm{W} 3+8,228 \times$ & \\
$\mathrm{W} 22+4,256 \times \mathrm{W} 32$ & \\
\hline
\end{tabular}

Tabela 3 - Modelos para a Estimativa das Características de Qualidade em Função dos Wi

A Função de Perda obtida aplicandose os dados do processo em questão (Tabelas 1, 2 e 3) é composta da combinação dos seis modelos individuais apresentados acima, mais as parcelas referentes à variabilidade de cada uma das características de qualidade. Assim, a mesma constitui-se de um grande número de termos, cada um deles representando somente uma pequena parte do modelo global.

Por esse motivo, estimou-se, através desta função, a perda associada a diversos ajustes dos fatores controláveis e, a partir destes pontos estimados, realizou-se uma nova Regressão Múltipla e obteve-se um novo modelo. Este novo modelo, cham ado metamodelo, possui os benefícios de ser um modelo mais compacto, mais fácil de tratar matematicamente, mantendo a representatividade do modelo original.

Metamodelo para a perda devida à má qualidade:

$$
\begin{aligned}
& \text { Perda }=5,634-1,1 . W 1+8,043 \cdot W 2+ \\
& 0,8536 . W 3+2,493 \cdot W 1 \cdot W 2 \\
& 1,804 \cdot W 1 . W 3-1,823 . W 2 \cdot W 3+ \\
& 1,513 . W 12+10,87 . W 22+2,31 . W 23(2)
\end{aligned}
$$


A equação acima fornece uma estimativa da Perda devido à má qualidade do produto; no entanto, essa perda está expressa adimensionalmente. Determinou-se, então, uma constante multiplicativa capaz de transformar esta perda adimensional em uma perda expressa em unidades monetárias (ou seja, em reais). A Figura 1 ilustra como é feita a determinação:

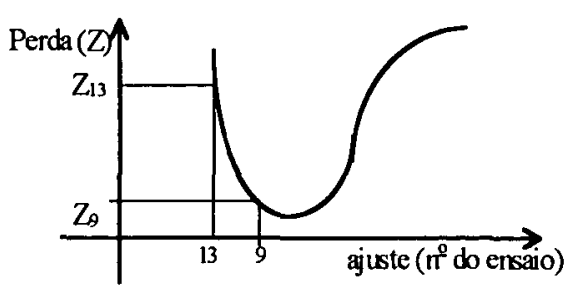

Figura 1 - Gráfico da Perda (devida à má qualidade).

Inicialmente, identificaram-se dois dos 20 ensaios realizados [Pasa, Ribeiro e Nunes, 1996] que apresentaram resultados extremos no que diz respeito à qualidade. A escolha recaiu sobre o ensaio $9(\mathrm{X} 1=24,01 \% ; \mathrm{X} 2=35,36 \% ; \mathrm{X} 3=$ $38,66 \%$ e X4 $=1,972 \%$ ), que representa um ajuste onde a qualidade do produto é boa, e o ensaio $13(\mathrm{X} 1=14,00 \% ; \mathrm{X} 2=$ $36,69 \% ; X 3=44,00 \%$ e X4 5,312\%), que representa um ajuste onde a qualidade do produto é ruim.

Através da Equação (2), estimou-se o valor adimensional da Perda devida à má qualidade para os ajustes respectivos a esses ensaios:
Perda adimensional (ajuste 9) = 5,004

Perda adimensional (ajuste 13) = 25,940

Utilizando o conhecimento que os engenheiros possuem a respeito do produto, foi possível atribuir um valor de mercado para os produtos obtidos em cada um desses ajustes:

Valor de mercado (ajuste 9) $\mathbf{P} \mathrm{RS}=$ 3,92

Valor de mercado (ajuste 13) $\mathbf{P} \mathrm{R} \$=$ 3,30

Diferença $=3,92-3,30=0,62$

Dessa forma, o problema pode ser equacionado da seguinte forma:

Perda em reais (ajuste 9) $=\mathrm{x}=$ $k(25,940)$

Perda em reais (ajuste 13 ) $=x+0,62$ $=\mathbf{k}(5,004)$

de onde se obtém:

$k=0,030$.

Determinada a constante $k$, pode-se escrever a Função de Perda (custos devidos à má qualidade) expressa em reais, multiplicando-se todos os termos da Equação (2) por $k$ :

$C Q=0,16902-0,0330 . \mathrm{W} 1+$
$0,24129 . \mathrm{W} 2+0,02561 . \mathrm{W} 3+$
$0,07479 . \mathrm{W} 1 . \mathrm{W} 2-0,05412 . W 1 . W 3-$
$0,05469 . W 2 . W 3+0,04539 . W 12+$
$0,3261 . W 22+0,0693 . W 23$ (3)




\section{PRODUÇÃO}

\subsection{Custos de Produção}

O modelo que fornece o custo de produção em função das variáveis independentes $W$ é apresentado a seguir. $\mathrm{O}$ mesmo foi obtido a partir de as informações fornecidas pelos engenheiros, baseadas diretamente no custo das matérias-primas em estudo $(\mathrm{X} 1, \mathrm{X} 2, \mathrm{X} 3$ e X4):

$$
\boldsymbol{C P}=1,64+0,045 . \mathrm{W} 1-0,04268 . \mathrm{W} 2
$$
$-0,05137 . W 3(4)$

\subsection{Custo Global da Mistura}

O custo global é composto pelo custo devido à má qualidade acrescido do custo de produção, ou seja pela soma das Equações (3) e (4):

$$
C G=1,79849+0,01344 . \mathrm{W} 1+
$$
0,19861 .W2 - 0,02576.W3 + 0,07479 .W1.W2 - 0,05412.W1.W3 -
$1,823 . W 2 . W 3+0,04539 . W 12+$

$0,3261 . W 22+0,0693 . W 23(5)$

\section{Otimização do Custo Global da Mistura}

Usando-se o modelo que descreve os custos devido à má qualidade (Equação 3 ), obtém-se 0 ajuste que minimiza esses custos. Esse ajuste aparece na Tabela 4.

Usando-se o modelo da Equação (4) para o custo de produção, obtém-se o ajuste que minimiza os custos de produção. Esse ajuste aparece descrito na Tabela 5.

Usando-se o modelo da Equação (5), determina-se o ajuste ótimo global, ou seja, aquele ajuste que minimiza a soma dos custos devido à má qualidade e de produção. Esse ajuste está apresentado

\begin{tabular}{|c|c|c|c|c|c|}
\hline Ajuste em W & Ajuste em X & $\begin{array}{l}\text { Características de } \\
\text { Qualidade }\end{array}$ & $\begin{array}{l}\text { Custos (R\$) } \\
\text { Produção }\end{array}$ & Global & Qualidade \\
\hline & & $Y 1=111,4$ & & & \\
\hline $\mathrm{W}_{1}=0,53$ & $X 1=23,31$ & $Y 2=64,3$ & 0,070 & 1,739 & 1,809 \\
\hline$W 2=-0,55$ & $X 2=40,82$ & $Y 3=325,9$ & & & \\
\hline \multirow[t]{3}{*}{$W 3=-1,00$} & $X 3=35,85$ & $Y 4=15760$ & & & \\
\hline & $X 4=0,00$ & $Y 5=95,2$ & & & \\
\hline & & $Y 6=172,8$ & & & \\
\hline
\end{tabular}
na Tabela 6.

Tabela 4 - Ajuste que minimiza os Custos devido à má Qualidade da mistura. 
PRODUÇÃO

\begin{tabular}{|l|l|l|l|l|l|}
\hline Ajuste em W & Ajuste em X & Caracteristicas de & \multicolumn{3}{|c|}{ Custos (R\$) } \\
& & Qualidade & \multicolumn{2}{|c|}{ Qualidade Produção Global } \\
\hline & & & & & \\
\hline $\mathrm{W} 1=-1,40$ & $\mathrm{X} 1=44,5$ & $\mathrm{Y} 2=66,3$ & 0,883 & 1,483 & 2,366 \\
\hline $\mathrm{W} 2=1,00$ & $\mathrm{X} 2=4,38$ & $\mathrm{Y} 3=359,8$ & & & \\
\hline $\mathrm{W} 3=1,00$ & $\mathrm{X} 3=43,8$ & $\mathrm{Y} 4=21130$ & & & \\
\hline & $\mathrm{X} 4=7,36$ & $\mathrm{Y} 5=96,0$ & & & \\
\hline & & $\mathrm{Y} 6=232,0$ & & & \\
\hline
\end{tabular}

Tabela 5 - Ajuste que minimiza os Custo de Produção

\begin{tabular}{|l|l|l|l|l|l|}
\hline Ajuste em W & Ajuste em X & $\begin{array}{l}\text { Características } \\
\text { deQualidade }\end{array}$ & \multicolumn{3}{|c|}{$\begin{array}{c}\text { Custos (R \$) } \\
\text { Qualidade Produção Global }\end{array}$} \\
\hline & & $\mathrm{Y} 1=112,5$ & & & \\
\hline $\mathrm{W} 1=0,67$ & $\mathrm{X} 1=15,79$ & $\mathrm{Y} 2=62,74$ & 0,111 & 1,634 & 1,745 \\
\hline $\mathrm{W} 2=-0,35$ & $\mathrm{X} 2=40,17$ & $\mathrm{Y} 3=342,3$ & & & \\
\hline $\mathrm{W} 3=1,00$ & $\mathrm{X} 3=36,72$ & $\mathrm{Y} 4=14380$ & & & \\
\hline & $\mathrm{X} 4=7,36$ & $\mathrm{Y} 5=102,8$ & & & \\
\hline & & $\mathrm{Y} 6=185,9$ & & & \\
\hline
\end{tabular}

Tabela 6 - Ajuste ótimo para o Custo Global 


\section{PRODUÇÃO}

A seguir, são apresentadas as curvas de isovalores obtidas a partir dos modelos para o Custo devido à má qualidade

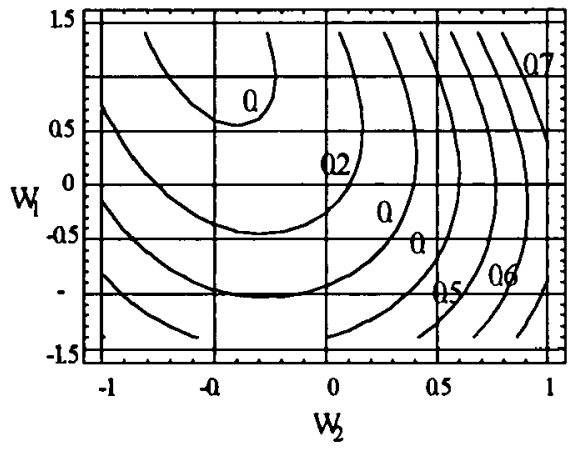

(a)
(Equação 3), Custo de produção (Equação 4) e Custo Global (Equação 5).

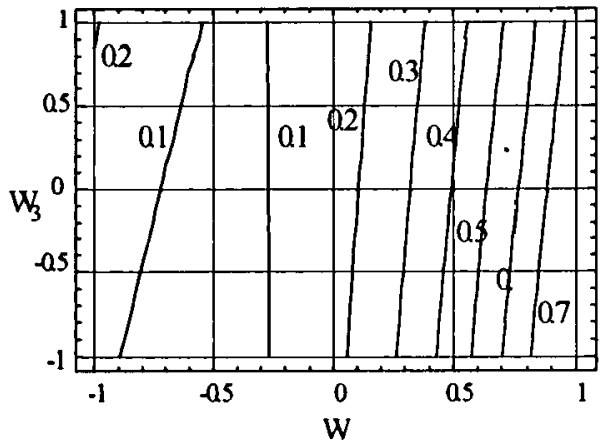

(b)

Figura 2 - Custo da má qualidade, quando (a) $W 3=1$ e (b) $W 1=0,67$.

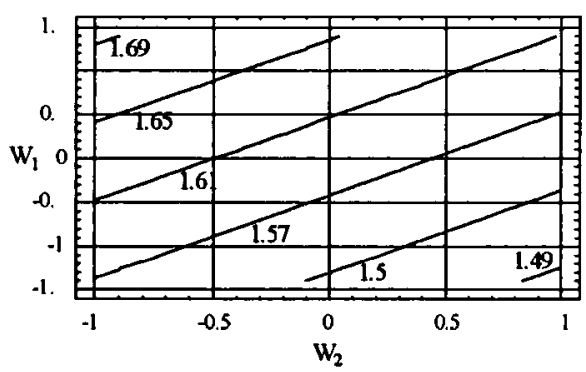

(a)

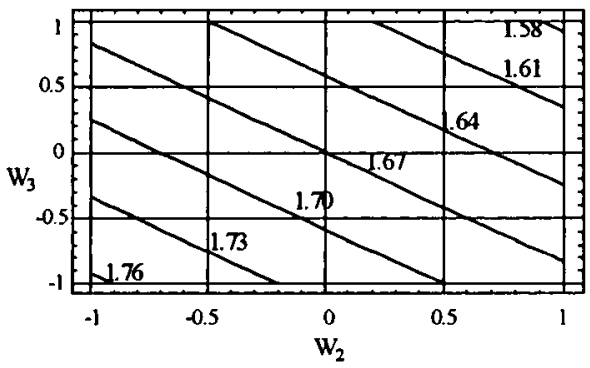

(b)

Figura 3 - Custo de Produção, quando (a) $W 3=1$ e (b) $W 1=0,67$. 


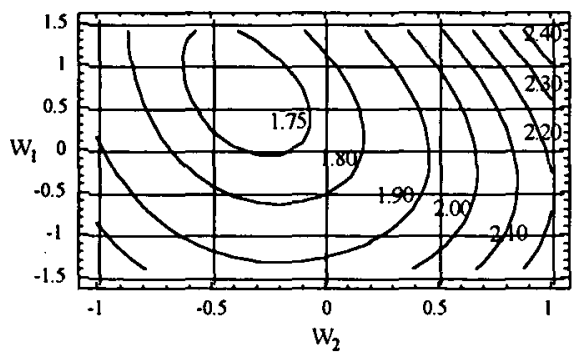

(a)

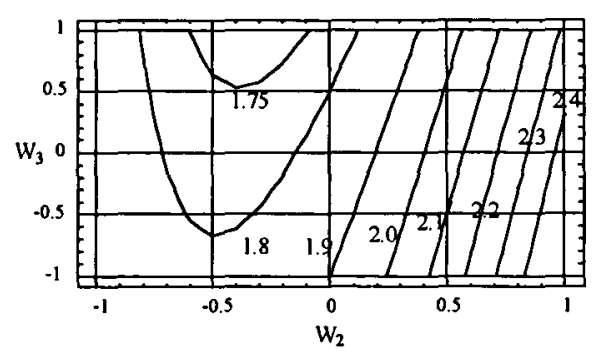

(b)

Figura 4 - Custo Global, quando (a) W3 = 1 e (b) W1 $=0,67$.

No item (a) das Figuras 2, 3 e 4, o valor de W3 é mantido constante em 1 (ajuste ótimo p/ o custo global) e Wl e W2 são variados. Isso quer dizer, em coordenadas $\mathrm{X}$, que o valor de $\mathrm{X} 4$ é mantido constante em $7,335 \%$ e Xl, X2 e X3 são variados.

No item (b) das mesmas figuras, o valor de Wl é mantido constante em 0,67 (ajuste ótimo p/ o custo global) e W2 e W3 são variados. Isso quer dizer, em coordenadas $\mathrm{X}$, que o valor da parcela (X2 $-0,568 . X 1)$ é mantido constante em $31 \%$ enquanto $\mathrm{X} 3$ e X4 são variados.
Na Figura 4.(a), por exemplo, a região interna à curva 1,75 corresponde a um conjunto de ajustes para W1 e W2 que mantêm o Custo Global inferior a $R \$ 1,75$. A partir da análise das Figura $4 a$ e $4 b$, e tendo o suporte da expressão para o Custo Global (Equação 5), é possível identificar uma região operacional, isto é, faixas de variação para W1, W2 e W3 que mantenham o Custo Global próximo do valor mínimo realizável. Essas faixas aparecem na Tabela 7.

Enquanto os percentuais dos componentes forem mantidos próximos destas quantidades, o Custo Global deve resultar igual ou inferior a $\mathrm{R} \$ \mathbf{1 , 7 5}$.

\begin{tabular}{|l|l|}
\hline Em variáveis independentes & \multicolumn{1}{|c|}{$\begin{array}{c}\text { Composição percentual da } \\
\text { mistura }\end{array}$} \\
\hline W1 entre 0,40 e 0,95 & X1 entre 12,5 e 18,0 \\
W2 entre $-0,50$ e $-0,20$ & X2 entre 37,0 e 43,5 \\
W3 entre 0,75 e 1,05 & X3 entre 36,0 e 37,5 \\
& X4 entre 6,5 e 7,5 \\
\hline
\end{tabular}

Tabela 7 - Ajustes ótimos para o Custo Global 


\section{PRODUÇÃO}

\section{Conclusões}

Este trabalho descreve um estudo de otimização dos custos de uma mistura de borracha, considerando-se duas parcelas fundamentais: os custos devido a má qualidade e os custos de produção. Este estudo dá continuidade ao trabalho apresentado anteriormente por Pasa, Ribeiro e Nunes (1996), onde havia sido feita a otimização exclusivamente da qualidade do produto.

Nos dois estudos, foram consideradas seis das características de qualidade de uma mistura de borracha: Abrasão, Densidade, Dureza, Tensão de ruptura, Módulo 300 e Alongamento. Os custos devido à má qualidade crescem na medida em que as características de qualidade do produto se afastam de seus valores alvo, enquanto que os custos de produção crescem na medida em que se definem misturas com percentuais elevados dos componentes mais caros.

Os custos devido à má qualidade foram modelados, usando-se a Função de Perda multivariada proposta por Ribeiro e Elsayed (1993, 1995), e os custos de produção foram representados por um modelo determinístico que considerava o custo de cada componente da mistura. $\mathrm{O}$ custo global foi obtido somando-se os custos devido à má qualidade e os custos de produção.

Obteve-se um modelo para o custo global e esse modelo foi usado como função objetivo, a ser minimizada no estudo de otimização.
O trabalho proporcionou importantes vantagens para a empresa, entre as quais se destacam:

- Expôs o corpo técnico da empresa a um estudo de otimização global, que considerou simultaneamente os aspectos referentes à qualidade e ao custo de produção de uma mistura.

- Obtiveram-se informações precisas a respeito dos custos devido à má qualidade e dos custos de produção, os quais foram expressos em unidades monetárias (reais);

- Os modelos obtidos permitem estimar esses custos (parciais e global) para uma mistura genérica; assim, caso, por motivos estratégicos, seja necessário abandonar o ótimo global para obter uma redução no custo de produção, ou uma melhoria na qualidade, os modelos e as figuras indicam qual o caminho a seguir.

- Obteve-se a indicação do ajuste ótimo global, que representa o melhor compromisso entre qualidade e custo de produção.

\section{Bibliografia}

Box, G. E P. \& Draper, N. (1987) Empirical Model Building and Response Surfaces. John Wiley and Sons, New York.

Caten, C.S. e Ribeiro, J.L. (1995) Custos da qualidade e da manufatura: Um estudo de caso na indústria química. Relatório Técnico 06/95, Programa de Pós Graduação em Engenharia de Produção/ UFRGS. Porto Alegre, RS. 
Caten, C.S. e Ribeiro, J.L. (1995b) Otimização experimental da qualidade: Massas cerâmicas e processo de fundição. Caderno Técnico 05/95, Programa de Pós Graduação em Engenharia de Produção/ UFRGS. Porto Alegre, RS.

CoRnell, J.A. (1981) Experiments with mixtures: designs, models, and the analysis of mixture data. John Wiley and Sons, New York.

Heinsman, J.A. \& Montgomery, D.C. (1995) Optimization of a household product formulation using a mixture experiment. Quality Engineering, v. 7, n. 3, p. 583-600.

Montgomery, D.C. AND Peck (1991) Introduction to linear regression analysis. John Wiley and Sons, New York, 2nd ed.

Ribeiro, J. L. \& Elsayed, E. A. (1995) A case study on process optimization using the gradient loss function. International Journal of Production Research, v. 33, n. 12, p. 3233-3248.

Pasa, G.S, Ribeiro, J.L. \& Nunes, D. B. (1996) Otimização da formulação de uma mistura - Parte 1: Qualidade. Revista Produção, v.6, n.1, p.65-82. 